\title{
Amygdala Changes Through Breathing Exercise in Coping with the Covid-19 Pandemic
}

\author{
Yuliana $^{1}$ \\ ${ }^{1}$ Medical Faculty of Udayana University, Indonesia
}

\begin{abstract}
The COVID-19 pandemic brings many psychological and physical changes. In coping with the COVID-19 pandemic, breathing exercise meditation is useful to calm the mind and body. Breathing exercise practice can change the brain, especially amygdala. This paper aims to give insight about amygdala changes through breathing exercise meditation in coping with the COVID-19 pandemic. This paper is aliterature review. Literature was identified from the archives from PubMed, Scopus, and Elsevier. The inclusion criteria were peer-reviewed paper. Keywords were amygdala, breathing, COVID-19, meditation, mindfulness. Results: Breathing exercise meditation and mindfulness practice can increase our awareness and reflections. In the long term, there will be changes in brain structure such as the amygdala. Conclusion: This paper is useful for psychology health knowledge. Patients, individuals, psychologists, physiotherapists, and psychiatrists can apply breathing exercise meditation and mindfulness methods for relaxing to prevent bad brain changes during the COVID-19 pandemic.
\end{abstract}

Keywords: Amygdala, Breathing, COVID-19, Meditation, Mindfulness

This is an open access article under the CC-BY-NC license.

\section{INTRODUCTION}

On December 31, 2019, there were some pneumonia cases with unknown origin in Wuhan, Hubei. Wuhan is located in the central-eastern of China. It was found that a new coronavirus (SARS-CoV-2) as the etiology. When this virus attacked humans, it can develop a severe pneumonia. The fatality rate was high for the elderly and patients with comorbid diseases. The disease is called as the coronavirus disease 2019. It is commonly called as the COVID-19 (De Giorgio, 2020). At first, the COVID-19 was thought of as a common respiratory infection. The symptoms were fever and cough. However, the symptoms became more severe such as respiratory distress, anosmia, cognitive dysfunction, defect of neuro-axonal integrity, brain, liver, heart, and kidney. At the later stage, there was cytokine storm (dysfunction of the immune system), thrombosis of multi-organ systems, and stroke withneurological deficits (Brann et al., 2020). Italy and many other countries in the world were attacked by the COVID-19 also. The COVID-19 became pandemic in March 2020 as stated by the World Health Organization (WHO). Until this paper was written, institutions and individuals are still struggling with the COVID-19 pandemic and all of its implications (De Giorgio, 2020).

The COVID-19 pandemic had many significant threats and impacts on our psychological life (Hagerty \& Williams, 2020). All of the humans in the world have opportunities to see this pandemic from many different points of view. People can use this special time to observe in detail what is going on. How we react as an individual is dependent on our characteristics and vulnerability. Our reaction is affected by our thinking. Emotions in coping with the COVID-19 can be various. They are fear, sad, frightened, anxious, restlessness, and uncertainty. The uncertain feeling is towards the government, the health care system, and the duration of the existing pandemic. Quarantine, 
lockdown, confinement, any mitigation forms, and social distancing will make anxiety and fear worsened (De Giorgio, 2020). Social mitigation during the COVID-19 pandemic will give a big threat to physical human connection, individual mental health, motivation, and brain profile in general (Hagerty \& Williams, 2020).

Social isolation during the COVID-19 pandemic had a tremendous impact on our daily life satisfaction. Going through distress and crisis need social support and group communities. Social isolation has a threat to survival. Social distancing can cause loneliness. Loneliness will make a psychological downward cycle, depress the immune systems, and cognitive function. In the severe form, it increases suicide rates. Decreasing social interaction will reduce memory performance and brain connectivity functions. People who have some social groups are less likely to develop depression. Social groups can be sports, religious, hobby, or charity groups. Social interactions increase the natural killer cell and endorphin systems. Meanwhile, there will be lower levels of blood pressure, body mass index, and C-reactive protein. On the other side, social isolation changes the prefrontal cortex function (Bzdok \& Dunbar, 2020).

Loneliness will cause underexpression of antiinflammatory-genes and overexpression of pro-inflammatory immune responses. Limited social stimulation is related to loneliness. In loneliness, there will be decreasing in the information processing (Bzdok \& Dunbar, 2020).

The ventromedial and dorsomedial prefrontal cortex, also striatal nucleus accumbens have a special role in social stimulation. The amygdala and hippocampus size are greater in people with larger social networks. They showed growth trajectories. The closer relation yields a more reactive amygdala. The amygdala lesion will diminish the sense of suitable private space with other people. The grey matter of the amygdala, the lower fiber tract integrity, the grey and white matter tissue has a smaller volume in social phobia. Social isolation lowers gene expression in the amygdala. The amygdala triggers the emotional reaction and regulates private physical distance (Bzdok \& Dunbar, 2020).

Social isolation and distancing have big impacts on the public. There will be changes in neurobiological architecture and functional brain. Antisocial behavior will develop. When social isolation happens on a large scale, there will disturb community stability and social cohesion. Loneliness for a long time may predispose to neurodegenerative diseases like Alzheimer's disease in the elderly. A longitudinal study of 332 adults showed there was improved empathy after being given regular training of cognitive skills. The improvement was in affective state and perspectivetaking. In this condition, the results revealed structural remodeling in the brain region, especially in the social brain network. The brain network involved is the frontoinsular network. When the training was given every day, there was thickening of the right anterior and middle of the insula. Compassion ratings were increased significantly. Joining the social club, singing (choirs), community, and hobby group was known to have an immediate effect on sense of the social engagement. Video conference in digital communication is very important to increase the social bonding between family and friends. Although they are in virtual space, the bonding is good. The visual component in video conference has an essential role in increasing social interaction (Bzdok \& Dunbar, 2020).

All people have their specific backgrounds. However, the experiences and feelings with the COVID-19 pandemic are very unique. The affected feelings might last for a long time and become maladaptive after the COVID-19 pandemic subsided. Pain, loss, grief, anxiety, and stress are all the feelings during the COVID-19 pandemic. A feeling has an emotional and neurobiological cascade. The cascade will let our body ready to respond and do an adaptation. Stress and fear will reduce brain function, mood regulation, decision making, and cognitive performance (W. D. Taylor \& Blackford, 2020). On the other side, positive emotion increases creativity (Spitzer, 2020). In the end, long term of negative feeling will change autonomic, endocrine, and immune system function in systemic physiology. Immune suppression, heart disease, and obesity will increase the risk for depression, post-traumatic stress disorder, and addiction (W. D. Taylor \& Blackford, 2020). 
Women tend to have an increased prevalence of post-traumatic stress disorder (PTSD) compared to men. There are sex differences of learned fear inhibition and medial prefrontal cortex function. Medial prefrontal cortex dysfunction can cause post-traumatic stress disorder. Fear generalization promotes defense mechanism in predicting the possible upcoming threat. Expressing fear excessively to insignificant stimuli can increase the possibility to develop posttraumatic stress disorder in the long term. Any disturbance in the inhibition of learned fear is the predisposition of post-traumatic stress disorder (Day et al., 2020).

Based on Maslow's Hierarchy, there is a hierarchy of psychological needs and selfactualization. There are five levels of human needs according to Maslow's hierarchy. The first level is physiological needs, including air and food. The second level is safety and comfort. The third level is love and possession. It includes emotional love with other people. The fourth level consists of evaluation and respect. The fifth level is self-actualization. During the COVID-19 pandemic, those five levels may be disrupted based on individual conditions. When the disruption is great, mental health will be burdened. The most often impact from the COVID-19 pandemic is human (social) connection. It includes in the third level of Maslow's hierarchy, i.e. love and belonging (possession). The physical isolation and social distancing are unsuitable with our basic needs as social beings (Hagerty \& Williams, 2020).

Sun et al. studied 2091 adult individuals in one month after the COVID-19 outbreak in Wuhan. They found the prevalence of post-traumatic stress disorder cases of the public in mainland China was 4.6\% (Sun et al., 2020). Liu et al. did a similar study of 285 respondents. They got a prevalence of $7 \%$ for post-traumatic stress disorders (Liu et al., 2020). Women and insomnia patients have a higher prevalence of post-traumatic stress disorders (Sun et al., 2020).

Post-traumatic stress disorders have a great impact on brain structure. There are changes in the fronto-limbic network. This will reduce emotion regulation. The volume of the hippocampus will decrease. Reactivity of the amygdala will higher (Guessoum et al., 2020).

The brain structures that correspond to our emotions are very complicated. The structures involved in fear and anxiety are the amygdala, ventromedial hypothalamus, hippocampus, and insular cortex. These structures undergo plasticity changes (W. D. Taylor \& Blackford, 2020). The fear is useful to protect human beings from dangerous situations. When we feel fear, we disrupt the circadian system. We can try to make some ways to save ourselves from dangerous conditions. Sometimes the danger hasn't come, but the fear and anxious feeling are needed to make people prepare for the future. Remember possible dangerous event might predispose our brain to some changes. Amygdala mediates fear and other emotions for learning and behavior. Fear is related to anxiety, panic, and substance disorders. The frontal brain area also controlled anxiety, panic, and fear. Frontal brain areas can minimize the emotional activation of other brain areas. Decision making has better result when anxiety is disappeared (De Giorgio, 2020).

The COVID-19 pandemic might have a catalyst role that exacerbates human connection deficits via various brain styles and pathways. These changes have profound effects on mental health consequences. There are three brain styles, i.e. "rumination" brain style, "anhedonia" brain style, and "threat dysregulation" brain style (Hagerty \& Williams, 2020).

"Rumination" brain style is often related to depression. It is repetitive distress as the source of the disorder. There is overwhelming brain connectivity regarding inner concerns and worries about the next moment in life. Meanwhile, it is too little connectivity for self-sensation. These unusual worries and concerns will affect how people see themselves. In the long term, the condition affects the relationship with others. Dependency, neediness, and aggressive behavior are negative characters of the "rumination" brain style. They tend to have less support from others due to social friction. "Rumination" brain style could exacerbate during the COVID-19 pandemic. Thus, the perceived threat might distort physical health (Hagerty \& Williams, 2020).

"Anhedonia" brain style is a lack of happiness from doing activities. It is not grateful for the brain's reward network. In anhedonia patients, there is less activation of the striatum. The reward system is under-activated. There is a dysfunctional reinforcement. People with anhedonia brain 
style tend to ignore rewards in social relationships. Right now, the COVID-19 pandemic is the trigger of anhedonia in vulnerable personality people. It is also known as blunted anticipatory characteristic. The motivation will be decreased. Anhedonia individual has a high risk of social withdrawal (Hagerty \& Williams, 2020).

Individuals with "threat dysregulation" brain style tends to have a bias in interpreting stimuli threat in their life. This is due to increased reactivity within the brain. Attentional bias is one of the symptoms of hyperactivation of the amygdala. There is less activity in the anterior cingulate and cortex. Social withdrawal and disconnection are reported in "threat dysregulation" brain-style individuals. During the COVID-19 pandemic, internal stimuli threats are our body signs and symptoms of any illness. Meanwhile, external stimuli threats are possible transmission from infected people and contaminated objects (Hagerty \& Williams, 2020).

The sympathetic activity has a higher level of adrenocortical release during this COVID-19 pandemic. There will be muscle vasoconstriction. Peripheral vascular resistance will be greater. Palpitation, tachycardia, and feelings of warmth and cold can cause overloading systems in the body. Due to this condition, sleep disturbance happens. It is similar to post-traumatic stress syndrome (Zhang et al., 2020).

Anxiety involves the reactions of the central nervous system, the prefrontal cortex, the motor region of the brainstem, the limbic and paralimbic structures. The paralimbic structures are the insula, amygdala, and the anterior cingulate gyrus. In normal conditions, the prefrontal cortex region is responsible for behavior, thought, and emotional regulation. It also inhibits unsuitable motor responses. In a stressful situation, the amygdala impairs the prefrontal cortex system. The amygdala activates the hypothalamus and brainstem pathway (Zhang et al., 2020).

The amygdala is critical in the processing of emotions. It is sensitized by acute stress. Acute stress will cause anxiety (Herrmann et al., 2020). However, the change of the amygdala is dynamic.

We can reduce the size of the amygdala by practicing mindfulness. It will reduce anxiety and depression. This practice is important for professionals, health workers, and populations as general. The reaction can be balanced. Understanding will be better when coping with the COVID19 pandemic situation. This practice can make the reactive amygdala smaller. Positive emotion contagion is sharing positive thoughts to deliver positive emotion to other people (De Giorgio, 2020). Emotions connect the brain and body. Emotions regulate what we perceive (Carlson, 2020)

When information about the COVID-19 pandemic overwhelmed our minds, our brain is difficult to proceed with that complicated information. Our brain tends to create its logical thinking to protect from fear and insecure feeling. This is a kind of defense mechanism. The mechanism might cause stereotypes. In this condition, people are easily influenced by hoax or fake information on social media. Vicious cycles about negative news can bombard our minds severely. The immune system will be down (De Giorgio, 2020). Social media has a double-edged sword. It can give virtual friendship, new information, and insight. However, on the other side, social media may bring hoax, social comparison, and unreal information. Increasing browsing time in social media and repeating message checking will increase levels of anxiety, fear, and psychological distress. The distress caused sleep disturbance (Keles et al., 2020).

Fear is a response to the upcoming threats. It is an adaptive response to fight for danger (Mertens et al., 2020). It helps in forming a suitable defense mechanism. The option can be fight or flight based on the signal. Fear is also a form of learning to anticipate a new situation. The amygdala is an important regulator of fear. There are special circuits in the basolateral part of the amygdala. It mediates fear learning and associated conditioned response. There is linking synaptic plasticity in the basolateral amygdala related to fear in the learning process (Sah et al., 2020). When the dangerous situation is uncertain and continuous such as during the COVID-19 pandemic, fear can turn into a maladaptive chronic burden. The manifestations can be the social phobia, xenophobia, and panic buying (Mertens et al., 2020).

It is crucial to investigate the reason for fear during the COVID-19 pandemic. Merten et al. identified the reasons for fear during the COVID-19 pandemic. From the online survey of 439 
respondents, the fear was evaluated by a special questionnaire. The fear of the Coronavirus questionnaire consisted of eight questions. The questions were eight questions about subjective worry, safety behavior, risk control, the risk for loved ones, and an open ended-question (Mertens et al., 2020).

Taylor et al. (2020) made the 36-item scale for the COVID-19 pandemic assessment. It was called the COVID Stress Scale (CSS). It consisted of five stress and anxiety-related factors in the United States and Canada. The factors were danger and contamination, fear about financial problems, xenophobia due to the COVID-19, repeated browsing, and traumatic symptoms (nightmare). Xenophobia during the COVID-19 pandemic means fear of foreigners that may transmit the infection (S. Taylor et al., 2020).

Lee et al. did a survey of 398 adult workers in the US. They used the Coronavirus Anxiety Scale (CAS) using an online survey. It was revealed that there was solid discrimination ability in functional impairment. This online survey tool is valid and practical in screening fear and anxiety related to coronavirus. The score of the survey was related to negative psychological emotion and wrong coping during the COVID-19 pandemic. The negative reactions about the COVID-19 were fear, alcohol use, negative religious practices, hopelessness, and suicidal idea. There was a different CAS score based on different sociodemographic and background (Lee et al., 2020).

There was a survey study of 120 medical staff who were fighting against COVID-19. The scores were high for somatization, anxiety, depression, and terror. The sleep quality was poor. Severe insomnia was $26.67 \%$. Maintaining a good sleep quality can stimulate the body to have the physical strength and a strong mental health state (Wu \& Wei, 2020).

Coping with the COVID-19 pandemic means to anticipate the potential threat. When the coping mechanism is activated, fear will be lower. Fear can fluctuate. It is a subjective concern. Fear and anxiety can shape behavior (Schimmenti et al., 2020). Reassurance, browsing appropriate news, identify emotional reactions, maintain social contacts, and stress-reducing activities are coping strategies (Wang et al., 2020).

Corona phobia is a phobia to anything connected to Coronavirus. Corona phobia may drive people to the hospital as soon as possible although they only catch a common cold or mild respiratory tract infection (Asmundson \& Taylor, 2020; De Giorgio, 2020). It is an emotional feeling that causes stress. Longer-term of corona phobia might be accompanied by cyberchondria. Cyberchondria is a repeated internet browsing for medical information due to worries about the physical health condition. Cyberchondria is related to anxiety. Increasing anxiety will lead to cyberchondria. Irrational belief system makes cyberchondria and corona phobia worsened. Therefore, browsing about coronavirus news should be balanced. We must browse positive and negative news about coronavirus equally. The more positive news is the better for our brain and mind (De Giorgio, 2020).

Meditation and mindfulness can improve sleeping for health workers, patients, and individuals in general. There is a specific method like mindfulness-based stress reduction. This system can improve anxiety and depression. In the long term, meditation and mindfulness practice can change the structural and functional of the brain. Meditation is a low cost. It can be done anywhere by everyone without any restrictions (Behan, 2020). Mindfulness and meditation can lower physiologic stress markers, depression, and anxiety (W. D. Taylor \& Blackford, 2020).

\section{METHODOLOGY}

This paper is a literature review. Literature was identified from the archives from PubMed, Scopus, and Elsevier. The inclusion criteria where the literature should be peer-reviewed and related to the topic of the paper. Keywords were amygdala, breathing, COVID-19, meditation, mindfulness. After careful screening based on title, abstract, and full text, there were 26 journals. From all of them, two journals from PubMed, 23 from Scopus (17 journals are Q1, 5 journals were Q2, one journal was Q3), and one from Elsevier. 
Journal selection was based on the journal contribution, looking for comparison and contrast among the journals, then giving synthesize and summary as the last step. Finally, the selected journals were put into a comprehensive narrative review.

\section{DISCUSSIONS}

In facing the COVID-19 pandemic situation, adaptation is a must. Every people have different problems and struggles. Coping and adaptation must be done every second. Patients with psychiatric disorders previously might feel depressed and overwhelmed. Children have to stay at home during online learning. Parents mostly must work from home. Finally, a house is a space for doing everything. In this restricted space, pressures may come from house members (Behan, 2020).

The financial burden and job losses during the COVID-19 pandemic make the situation more difficult (Behan, 2020; Hagerty \& Williams, 2020). Parents have to struggle for financial stability. Children have to remain at home during the COVID-19 pandemic. Domestic violence would happen if the parents' mind is not well controlled. Meditation and mindfulness give hope in calming complicated minds during this hard time (Behan, 2020).

Fear has three phases, i.e., acquisition, storage, and retrieval. Acquisitions are sensory input and conditioning. Storage is the forming of memory. Retrieval is the initiation of a defense mechanism (Sah et al., 2020).

Schimmenti et al. (2020) analyzed four reasons for fear. The reasons are fear of the body, fear of anything happens to other people with interpersonal relationships, fear of not knowing anything, and fear of not taking the right action. Fear is related to idiosyncrasies. The most common anxiety and fear during the COVID-19 pandemic is health anxiety. Fear about infecting our loved ones is a bad feeling of all. Uncertainty with the current pandemic condition will increase fear and anxiety. Hypervigilant about any changes in the body will induce fear and anxiety. Any unusual feeling about the body is not permanent. Fear about knowing something can lead to inaction due to afraid of risk taking-behavior (Schimmenti et al., 2020).

The stress response is arranged by the autonomic nervous system (ANS) and the hypothalamic-pituitary-adrenal (HPA) axis. This axis is important in stress neurobiology. It has a critical role in social isolation (Bzdok \& Dunbar, 2020). The hypothalamus and brainstem are the most important brain regions in stress responses. The corticotropin-releasing factor is released at a higher level. Changes in neuroanatomy and neuroendocrine during the stress condition can make the immune systems weak. The inflammatory factors, such as tumor necrosis factor-a (TNF-a), interleukin (IL)-1b, IL-6, and interferon-g will be higher. This will increase the risk of autoimmune or inflammatory diseases (Wu \& Wei, 2020). Noradrenaline is increased during the stress period. This will sensitize the amygdala (Herrmann et al., 2020).

The amygdala is sensitized by acute stress. The specificity of the amygdala will diminish; thus, it becomes more difficult to differentiate between stimuli. In adverse circumstances, the acknowledgment of every potential risk may be beneficial. The basolateral amygdala complex, the insula, the prefrontal cortex, and the anterior cingulate cortex mediate stress and emotional effects on memory consolidation. Memory consolidation of the COVID-19 pandemic could lead to fearful memories. Fear in long term can cause anxiety disorder. Finally, there will be increasing spine numbers in the amygdala to add connectivity of synapses. Decreasing stress will change the morphological pattern of the amygdala. Reducing stress will also decrease the grey matter. The left amygdala was more activated during a fearful situation. It means there are lateralization effects of the amygdala when there is any stressor. The left amygdala has more frequent activation compared to the right one in trauma conditions such as post-traumatic stress disorders (Herrmann et al., 2020).

The amygdala is a region in the middle of the temporal pole, associated with fear learning mediation. The amygdala is the emotional center of the brain. The amygdala is made of a group of nuclei. It receives input from the cortex and sub-cortex parts of the brain. There is a lot of internuclear activity in the amygdala, especially in the basolateral amygdala and central amygdala. 
The basolateral amygdala forms the main input, meanwhile, the output structure is formed by the central amygdala. Information is kept in the basolateral amygdala. This action will result in a memory trace. Projection from the basolateral amygdala to the central amygdala arises a defensive mechanism (Sah et al., 2020).

The basolateral amygdala is in the middle of the temporal pole. It consists of the lateral and basal nuclei. The lateral part is at the dorsal side of the basal nuclei. The lateral nuclei are divided into dorsolateral, ventrolateral, and ventromedial nuclei. Meanwhile, the basal nuclei are formed by the basolateral nucleus and the basomedial nuclei (accessory basal nuclei). Their divisions have distinct cytoarchitectonic and connection. The basolateral nucleus consists of the rostral magnocellular, caudal intermediate, and parvicellular subdivisions. The accessory basal nuclei have magnocellular subdivision and mediocaudal parvicellular subdivision (Sah et al., 2020).

When people feel that the danger is incoming, there will be several internal psychological responses. The responses include emotional, behavior, and thinking changes. Fear, anxiety, grief, depression will happen. Physiological reactions may vary, i.e. pain, palpitation, chest tightness, and fatigue. Doing physical and mental exercise can overcome post-traumatic stress disorder (Wu \& Wei, 2020).

Strengthening psychological health can be done in many ways. Those ways are increasing appraisal of the body, secure attachment, regulation of emotion, acceptance, thinking positive things, and responsibility. Appraisal of the body means to evaluate the body condition honestly without any subjectivity. Improving posture, doing physical, and mental exercise (mindfulness practice) can reduce fear and increase safety. Acceptance is flexible, receptive, and open to any experience. Meditation and mindfulness stimulate acceptance and self-compassion (Schimmenti et al., 2020).

Mild pain, mental problems, and somatic conditions can be relieved by meditation and mindfulness. However, these heterogeneous conditions are difficult to be researched in a systematic system. Mindfulness-based cognitive therapy and mindfulness-based stress reduction are formal meditation practices. The practices consist of eight-week programs for two hours duration each week. Mindfulness-based stress reduction was developed by John Kabat-Zinn in the 1970s. Mindfulness-based cognitive therapy is more general. It is focused on a combination of cognitive and mindfulness methods (Behan, 2020).

Meditation is different from mindfulness. Meditation is intended to calm the mind and increase awareness of ourselves, the environment, and our minds. Meditation has been used as a routine practice to calm the mind all over the world by different cultural backgrounds. Moreover, meditation is used as one of the therapy methods for psychological problems. Mindfulness is being aware of every second of the present situation. Meditation practice includes mindfulness of breathing (especially diaphragmatic breathing), walking meditation, the compassion of love, mantra, or other simple repeated activities (Behan, 2020). Emotion can be decoded from the body posture (Spitzer, 2020). That's why any mindfulness and meditation practice by using the body gesture (breathing, walking, or others) may stimulate positive feelings (Behan, 2020).

Breathing in meditation practice is done by inhaling deeply to expand the lungs downward. The inhaling process is by the nose, then do a pause before exhaling slowly by the mouth. Counting is done while breathing slowly. It is important to maintain the respiratory rate between six to eight breaths per minute. Inhale is 5 second's count. Exhale is also 5 second's count. There is a pause between inhale and exhale. Ten-minutes sessions of diaphragmatic breathing can reduce stress, blood pressure, and cortisol level in the saliva in a preliminary experimental study. Even 30 to 60 seconds of deep breathing before doing any stressful activities can make big difference in the level of perceived pressure. This practice of breathing is useful as a prevention tool. When the stress, fear, and anxiety are too heavy, medical attention such as a psychologist or psychiatrist should be contacted (Hopper et al., 2019).

Mindfulness can be implemented while doing simple daily activities such as waiting for a red light in the traffic, washing hands, or using hand sanitizer. Mindfulness is a wellness self-check 
to increase energy levels. One-minute mindfulness each time is enough. Compassion and love meditation means to visualize the presence of empathy and calmness to the present moment. In meditation, past and future moments need to be put away for a while. Naming the arising feeling or emotion is also self-awareness. By naming the emotion, it is easier to tame it (Behan, 2020).

Meditation means let go of anything in the past. Being aware of the present moment will arise a new perspective. Observing the flow of thought is useful for remaining calm and aware. Our mind is like a huge complicated network. We need to sort the important thing. Breath is used as the anchor for the thought and present moment. A wandering mind is an unhappy mind. Body scanning from the head until toe can be used as an anchor also. This type of body scan is different from the scanner machine for imaging in hospitals. Body scanning in the meditation methods means being aware of all parts of the body from the most upper part (the edge of the hair) until the most lower part (the toes). The goal of meditation is to make someone spend the time in every moment calmly. Long-term meditation will have a better effect on reducing stress and anxiety. There is increasing activity in the cingulate cortex, the prefrontal cortex, and the hippocampus. Meanwhile, the amygdala had decreasing activity. It results in better emotional regulation (Behan, 2020).

The most common negative emotional feelings during the COVID-19 pandemic are anxious, despair, fear, and overwhelm. These negative feelings are natural for this condition. The feelings can remain for an uncertain duration although the COVID-19 pandemic has ended. Therefore, practicing mindfulness is very essential at this moment. Post-traumatic stress disorders can be relieved by this method. Stress, cortisol level, and blood pressure can be reduced through the regular practice of mindfulness (Singh \& Hwang, 2019).

Mindfulness and meditation can be learned online. These techniques are easy to be done by children, teens, adults, elderly, and even disabled people. There are many applications available for online meditation such as Calm, Insight Timer, and Headspace. In the short term, sleep quality will increase by practicing mindfulness and meditation (Zheng et al., 2020).

There are some organizations for meditation practice such as the Royal College of Physicians (\#pauseforapoem). The Sanctuary, and also the Dublin Buddhist Centre. They offer online learning meditation for a free or discounted price. There is a lot of demand for such courses. This is a good sign for coping with the COVID-19 pandemic. It is a huge area to be studied (Behan, 2020).

In every crisis, it was shown that the eternal thing is the change. The COVID-19 pandemic will be ended although it is not unsure when the time is going to be. Meditation and mindfulness provide shelter for people during this insecure time. The regular practice of mindfulness and meditation must be taken by all people to increase awareness during this hard time. This will bring benefits to ourselves and our society. Anything shall pass is the suitable motto for coping with the COVID-19 pandemic in a mindfulness way (Behan, 2020).

\section{CONCLUSION}

The COVID-19 pandemic has many effects on the biological and behavioral parts of human life. In the biological aspect, the COVID-19 pandemic affects the human brain, especially the limbic system and the amygdala. In behavioral parts, it affects mental health and psychology. Fear will disturb the amygdala in structurally and functionally. Mindfulness and meditation can soothe fear and other negative feelings. Fear of being infected and spread the virus to others cause anxiety and corona phobia. Mindfulness and meditation can be done in simple ways such as breathing practice, walking, and realizing the present moment.

\section{LIMITATION \& FURTHER RESEARCH}

The limitations of the study are lack of recent studies about the effects of breathing exercise to amygdala changes during the COVID-19 pandemic. Further research should be done to extend the characteristics of amygdala changes and other meditation methods besides breathing. 


\section{REFERENCES}

Asmundson, G. J. G., \& Taylor, S. (2020). Coronaphobia: Fear and the 2019-nCoV outbreak. Journal of Anxiety Disorders, 70. https://doi.org/10.1016/j.janxdis.2020.102196

Behan, C. (2020). The benefits of Meditation and Mindfulness practices during times of crisis such as Covid-19. Irish Journal of Psychological Medicine, 3-5. https://doi.org/10.1017/ipm.2020.38

Brann, D. H., Tsukahara, T., Weinreb, C., Lipovsek, M., Berge, K. Van Den, Gong, B., ... Grubb, M. S. (2020). Non-neuronal expression of SARS-CoV-2 entry genes in the olfactory system suggests mechanisms underlying COVID-19 - associated anosmia. Science Advance, 6(July), 1-20.

Bzdok, D., \& Dunbar, R. I. M. (2020). The Neurobiology of Social Distance. Trends in Cognitive Sciences, 24(9), 717-733. https://doi.org/10.1016/j.tics.2020.05.016

Carlson, E. R. (2020). COVID-19 and Educational Engagement. Journal of Oral and Maxillofacial Surgery, 78(7), 1049-1051. https://doi.org/10.1016/j.joms.2020.04.033

Day, H. L. L., Suwansawang, S., Halliday, D. M., \& Stevenson, C. W. (2020). Sex differences in auditory fear discrimination are associated with altered medial prefrontal cortex function. Scientific Reports, 10(1), 1-10. https://doi.org/10.1038/s41598-020-63405-w

De Giorgio, A. (2020). Global Psychological Implications of Severe Acute Respiratory Syndrome Coronavirus 2 (SARS-CoV-2) and Coronavirus Disease-2019 (COVID-19). What Can Be Learned From Italy. Reflections, Perspectives, Opportunities. Frontiers in Psychology, 11(July), 1-6. https://doi.org/10.3389/fpsyg.2020.01836

Guessoum, S. B., Lachal, J., Radjack, R., Carretier, E., Minassian, S., Benoit, L., \& Moro, M. R. (2020). Adolescent psychiatric disorders during the COVID-19 pandemic and lockdown. Psychiatry Research, 291(January). https://doi.org/10.1016/j.psychres.2020.113264

Hagerty, S. L., \& Williams, L. M. (2020). The impact of COVID-19 on mental health: The interactive roles of brain biotypes and human connection. Brain, Behavior, \& Immunity - Health, 5(January), 100078. https://doi.org/10.1016/j.bbih.2020.100078

Herrmann, L., Vicheva, P., Kasties, V., Danyeli, L. V., Szycik, G. R., Denzel, D., ... Walter, M. (2020). fMRI Revealed Reduced Amygdala Activation after Nx4 in Mildly to Moderately Stressed Healthy Volunteers in a Randomized, Placebo-Controlled, Cross-Over Trial. Scientific Reports, 10(1), 1-14. https://doi.org/10.1038/s41598-020-60392-w

Hopper, S. I., Murray, S. L., Ferrara, L. R., \& Singleton, J. K. (2019). Effectiveness of diaphragmatic breathing for reducing physiological and psychological stress in adults : a quantitative systematic review. https://doi.org/10.11124/JBISRIR-2017-003848

Keles, B., Mccrae, N., \& Grealish, A. (2020). A systematic review : the influence of social media on depression, anxiety and psychological distress in adolescents. International Journal of Adolescence and Youth, 25(1), 79-93. https://doi.org/10.1080/02673843.2019.1590851

Lee, S. A., Mathis, A. A., Jobe, M. C., \& Pappalardo, E. A. (2020). Clinically significant fear and anxiety of COVID-19: A psychometric examination of the Coronavirus Anxiety Scale. Psychiatry Research, 290. https://doi.org/10.1016/j.psychres.2020.113112.

Liu, N., Zhang, F., Wei, C., Jia, Y., Shang, Z., \& Sun, L. (2020). Prevalence and predictors of PTSS during COVID-19 outbreak in China hardest-hit areas: Gender differences matter. Psychiatry Research, 287. https://doi.org/10.1016/j.psychres.2020.112921

Mertens, G., Gerritsen, L., Duijndam, S., Salemink, E., \& Engelhard, I. M. (2020). Fear of the coronavirus (COVID-19): Predictors in an online study conducted in March 2020. Journal of Anxiety Disorders, 74, 102258. https://doi.org/10.1016/j.janxdis.2020.102258

Sah, P., Sun, Y., \& Gooch, H. (2020). Fear conditioning and the basolateral amygdala. F1000Research, 9, 1-8. https://doi.org/10.12688/f1000research.21201.1

Schimmenti, A., Billieux, J., \& Starcevic, V. (2020). The four horsemen of fear during the COVID 
pandemic. Clinical Neuropsychiatry, 17(2), 41-45. https://doi.org/10.36131/CN20200202

Singh, N. N., \& Hwang, Y. (2019). Mindfulness-based programs and practices for people with intellectual and developmental disability. https://doi.org/10.1097/YCO.0000000000000570

Spitzer, M. (2020). Masked education? The benefits and burdens of wearing face masks in schools during the current Corona pandemic. Trends in Neuroscience and Education, 20(100138). https://doi.org/10.1016/j.tine.2020.100138.

Sun, L., Sun, Z., Wu, L., Zhu, Z., Zhang, F., Shang, Z., ... Liu, W. (2020). Prevalence and Risk Factors of Acute Posttraumatic Stress Symptoms during the COVID-19 Outbreak in Wuhan, China. MedRxiv. https://doi.org/10.1101/2020.03.06.20032425.

Taylor, S., Landry, C. A., Paluszek, M. M., Fergus, T. A., Mckay, D., \& Asmundson, G. J. G. (2020). Development and initial validation of the COVID Stress Scales. Journal of Anxiety Disorders, 72(April), 102232. https://doi.org/10.1016/j.janxdis.2020.102232

Taylor, W. D., \& Blackford, J. U. (2020). Mental Health Treatment for Front-Line Clinicians During and After the Coronavirus Disease 2019 (COVID-19) Pandemic: A Plea to the Medical Community. Annals of Internal Medicine, 2019(10), 19-21. https://doi.org/10.7326/m202440

Wang, G., Zhang, Y., Zhao, J., Zhang, J., \& Jiang, F. (2020). Mitigate the effects of home confinement on children during the COVID-19 outbreak. The Lancet, 395(10228), 945-947. https://doi.org/10.1016/S0140-6736(20)30547-X

Wu, K., \& Wei, X. (2020). Analysis of Psychological and Sleep Status and Exercise Rehabilitation of Front-Line Clinical Staff in the Fight Against COVID-19 in China. Medical Science Monitor Basic Research, 26, e924085. https://doi.org/10.12659/MSMBR.924085

Zhang, J. jin, Dong, X., Cao, Y. yuan, Yuan, Y. dong, Yang, Y. bin, Yan, Y. qin, Akdis, C. A., \& Gao, Y. dong. (2020). Clinical characteristics of 140 patients infected with SARS-CoV-2 in Wuhan, China. Allergy: European Journal of Allergy and Clinical Immunology, 75(7), 1730-1741. https://doi.org/10.1111/all.14238

Zheng, A. M. X., Yao, J., \& Narayanan, J. (2020). Mindfulness Buffers the Impact of COVID-19 Outbreak Information on Sleep Duration. PsyArXiv, 20(3). doi: 10.31234/osf.io/wuh94 